\title{
An Efficient Computation of Effective Ground Range Using an Oblate Earth Model
}

\author{
Dalal A. Maturi, ${ }^{1}$ Malik Zaka Ullah, ${ }^{1}$ Shahid Ahmad, ${ }^{2}$ and Fayyaz Ahmad ${ }^{3}$ \\ ${ }^{1}$ Department of Mathematics, King Abdulaziz University, Jeddah 21589, Saudi Arabia \\ ${ }^{2}$ Department of Mathematics, Government College University, Lahore 54000, Pakistan \\ ${ }^{3}$ Department of Mathematics, Government College University, Faisalabad 38000, Pakistan \\ Correspondence should be addressed to Malik Zaka Ullah; mzhussain@kau.edu.sa
}

Received 12 May 2014; Revised 22 July 2014; Accepted 4 August 2014; Published 27 August 2014

Academic Editor: Dumitru Baleanu

Copyright (C) 2014 Dalal A. Maturi et al. This is an open access article distributed under the Creative Commons Attribution License, which permits unrestricted use, distribution, and reproduction in any medium, provided the original work is properly cited.

An effcient method is presented to calculate the ground range of a ballistic missile trajectory on a nonrotating Earth. The spherical Earth model does not provide good approximation of distance between two locations on the surface of Earth. We used oblate spheroid Earth model because it provides better approximations. The effective ground range of a ballistic missile is an arc-length of a planner elliptic (or circle) curve which passes through the launch and target points on the surface of Earth model. A general formulation is presented to calculate the arc-length of an elliptic (or circle) curve which is the intersection of oblate Earth model and a plane. Explicit formulas are developed to calculate the coordinates of center of the ellipse as well as major and minor axes which are necessary ingredients for the calculation of effective ground range.

\section{Introduction}

We developed a method to calculate the distance between two points constrained to lie on the surface of the oblate spheroid. We assume the definition of distance between launch and target points (the launch and target points are just two given arbitrary points) to be the length of the curve resulting from the intersection of the given oblate spheroid with a plane which passes through the launch point and the target point. There are infinite numbers of planes which pass through the launch and target points, for example, (1) the plane which passes through the normal at launch point (trajectory plane) and (2) the plane which passes through the geometrical center of the oblate spheroid (geocentric plane). All these planes can be obtained from a single plane by rotating it about the line joining the launch and target points. Therefore, we take a general plane which passes through the two given points on the oblate spheroid. We see that the trace of the oblate spheroid in the general plane is an ellipse. We find the semimajor axis, semiminor axis, and center of the ellipse. We also find the unit vector along the major axis of the ellipse. By calculating the position vectors of the launch and target point with respect to the center of the ellipse, we calculate the angles which these vectors make with major axis of the ellipse. Finally, we find the smaller arc length of the ellipse between these two angles which is the surface range between the given two points.

Many researchers have investigated numerical methods related to ballistic missiles and satellite launch vehicles. In [1], the authors discussed computation of the different errors in the ballistic missiles range. Estimation and prediction of ballistic missiles are discussed in [2]. Some recent research work about ballistic missiles and satellite launch vehicles can be found in [3-18]. Escobal [19] and Nguyen and Dixson [20] have formulated the problem to calculate range of a ballistic missile over the none-rotating oblate spheroid Earth model. Both authors define the elliptic curve over the surface of oblate Earth model by the intersection of a plane which passes through the launch, target points, and center of the oblate spheroid. Clearly in this case, the center of the ellipse (if the cutting plane is parallel to equatorial plane then resulting intersecting curve is a circle.) is the center of oblate spheroid. Once they know the center, it is easy to use rotational transformation matrix to calculate the orientation of major 
and minor axis. But in reality, the trajectory plane of ballistic missile passes through the normal vector at launch (or target) point to the surface of Earth model and the target point (or launch point). It is not necessarily true that trajectory plane passes through the center of oblate spheroid. It means, we require a general formulation of the problem to calculate the elements of general intersecting ellipse. Figure 1 shows two intersecting ellipses passing through launch and target points.

\section{Trace of the Oblate Spheroid in the Plane}

General equation of a plane is given by

$$
n_{1} x+n_{2} y+n_{3} z=d
$$

where $\vec{n}=\left[n_{1}, n_{2}, n_{3}\right]$ is a vector normal to the plane and $d$ is the distance of the plane from the origin of coordinates. The equation of the oblate spheroid can be written as

$$
\frac{x^{2}+y^{2}}{a^{2}}+\frac{z^{2}}{b^{2}}=1,
$$

where $a$ is the semimajor axis and $b$ is the semiminor axis of the oblate spheroid. The trace of the oblate spheroid (2) in the plane (1) is calculated as follows. In (1), for $n_{3} \neq 0$ we have

$$
z=\frac{d-n_{1} x-n_{2} y}{n_{3}} .
$$

If $n_{3}=0$, then $\vec{n}$ is parallel to the equatorial plane, that is, $\widehat{n} \perp$ polar axis $(0,0,1)$. Substituting this value of $z$ in (2)

$$
\begin{gathered}
\frac{x^{2}+y^{2}}{a^{2}}+\frac{1}{b^{2} n_{3}^{2}}\left(d^{2}+n_{1}^{2} x^{2}+n_{2}^{2} y^{2}-2 d n_{1} x\right. \\
\left.-2 d n_{2} y+2 n_{1} n_{2} x y\right)=1, \\
b^{2} n_{3}^{2}\left(x^{2}+y^{2}\right)+a^{2}\left(n_{1}^{2} x^{2}+n_{2}^{2} y^{2}+d^{2}-2 d n_{1} x\right. \\
\left.-2 d n_{2} y+2 n_{1} n_{2} x y\right)=a^{2} b^{2} n_{3}^{2}, \\
\left(a^{2} n_{1}^{2}+b^{2} n_{3}^{2}\right) x^{2}+\left(a^{2} n_{2}^{2}+b^{2} n_{3}^{2}\right) y^{2}+2 a^{2} n_{1} n_{2} x y \\
-2 d n_{1} a^{2} x-2 d n_{2} a^{2} y-a^{2} b^{2} n_{3}^{2}+a^{2} d^{2}=0,
\end{gathered}
$$

which is the equation of a conic. We know that a general equation of a conic is

$$
a x^{2}+b y^{2}+2 g x+2 f y+2 h x y+c=0 .
$$

This equation represents an ellipse or a circle if its discriminant $h^{2}-a b<0$. The discriminant of (5) is

$$
\begin{aligned}
& \left(a^{2} n_{1} n_{2}\right)^{2}-\left(a^{2} n_{1}^{2}+b^{2} n_{3}^{2}\right)\left(a^{2} n_{2}^{2}+b^{2} n_{3}^{2}\right) \\
& =a^{4} n_{1}^{2} n_{2}^{2}-a^{4} n_{1}^{2} n_{2}^{2}-a^{2} b^{2} n_{1}^{2} n_{23}^{2}-a^{2} b^{2} n_{2}^{2} n_{3}^{2}-b^{4} n_{3}^{4} \\
& =-b^{2} n_{3}^{2}\left(a^{2} n_{1}^{2}+a^{2} n_{2}^{2}+b^{2} n_{3}^{2}\right),
\end{aligned}
$$

which is always less than zero. Hence, (5) represents an ellipse or a circle. Thus, we see that the trace of the oblate spheroid (given by (2)) in the plane (given by (1)) is an ellipse.

\section{Center of the Ellipse}

Let $\vec{s}=\left[s_{1}, s_{2}, s_{3}\right]$ be the position vector of the center of the ellipse. If $P_{1}\left(\vec{r}_{1}\right)$ is any point on the ellipse, then $\vec{r}_{1}$ can be parametrized as

$$
\begin{aligned}
& \vec{r}_{1}=\vec{s}+\mu \widehat{e}, \\
& \vec{r}_{1}=\left[s_{1}+\mu e_{1}+s_{2}+\mu e_{2}, s_{3}+\mu e_{3}\right],
\end{aligned}
$$

where $\vec{e}=\left[e_{1}, e_{2}, e_{3}\right]$. Since point $P_{1}$ also lies on the oblate spheroid, it must satisfy (2)

$$
\begin{gathered}
\frac{\left(s_{1}+\mu e_{1}\right)^{2}+\left(s_{2}+\mu e_{2}\right)^{2}}{a^{2}}+\frac{\left(s_{3}+\mu e_{3}\right)^{2}}{b^{2}}=1 \\
b^{2}\left[s_{1}^{2}+s_{2}^{2}+\mu^{2}\left(e_{1}^{2}+e_{2}^{2}\right)+2 \mu\left(e_{1} s_{1}+e_{2} s_{2}\right)\right] \\
+a^{2}\left(s_{3}^{2}+\mu^{2} e_{3}^{2}+2 \mu s_{3} e_{3}\right)=a^{2} b^{2} \\
\left(b^{2}\left(e_{1}^{2}+e_{2}^{2}\right)+a^{2} e_{3}^{2}\right) \mu^{2}+2\left(b^{2}\left(e_{1} s_{1}+e_{2} s_{2}\right)+a^{2} e_{3} s_{3}\right) \mu \\
+b^{2}\left(s_{1}^{2}+s_{2}^{2}\right)+a^{2} s_{3}^{2}-a^{2} b^{2}=0 .
\end{gathered}
$$

Equation (10) is quadratic in $\mu$. If $P_{1}\left(\vec{r}_{1}\right)$ is any point on the ellipse, then there must be another point $P_{1}^{\prime}\left(\vec{v}_{1}^{\prime}\right)$ on the ellipse such that

$$
\vec{v}_{1}^{\prime}=\vec{s}-\mu \widehat{e} .
$$

Thus, (10) should give us two values of $\mu$, both having the same magnitude but opposite in sign. It means that coefficient of $\mu$ in (10) should be zero:

$$
b^{2}\left(e_{1} s_{1}+e_{2} s_{2}\right)=-a^{2} e_{3} s_{3} .
$$

Let $P_{2}\left(\vec{r}_{2}\right)$ be another point on the ellipse such that

$$
\vec{r}_{2}=\vec{s}+\eta \widehat{q}
$$

and $\widehat{q}$ is perpendicular to $\widehat{e}$. Doing the same steps as done for (12), we have

$$
b^{2}\left(q_{1} s_{1}+q_{2} s_{2}\right)=-a^{2} q_{3} s_{3} .
$$

Dividing (14) by (12), we have

$$
\frac{q_{1} s_{1}+q_{2} s_{2}}{e_{1} s_{1}+e_{2} s_{2}}=\frac{q_{3}}{e_{3}},
$$

which can be written as

$$
s_{1}\left(e_{3} q_{1}-q_{3} e_{1}\right)=s_{2}\left(e_{2} q_{3}-e_{3} q_{2}\right) .
$$

Since $\hat{q}$ and $\hat{e}$ lay in the plane of the ellipse, therefore we can write

$$
\begin{aligned}
& \hat{n}=\widehat{e} \times \widehat{q}, \\
& n_{1}=e_{2} q_{3}-e_{3} q_{2}, \\
& n_{2}=e_{3} q_{1}-q_{3} e_{1}, \\
& n_{3}=e_{1} q_{2}-e_{2} q_{1} .
\end{aligned}
$$




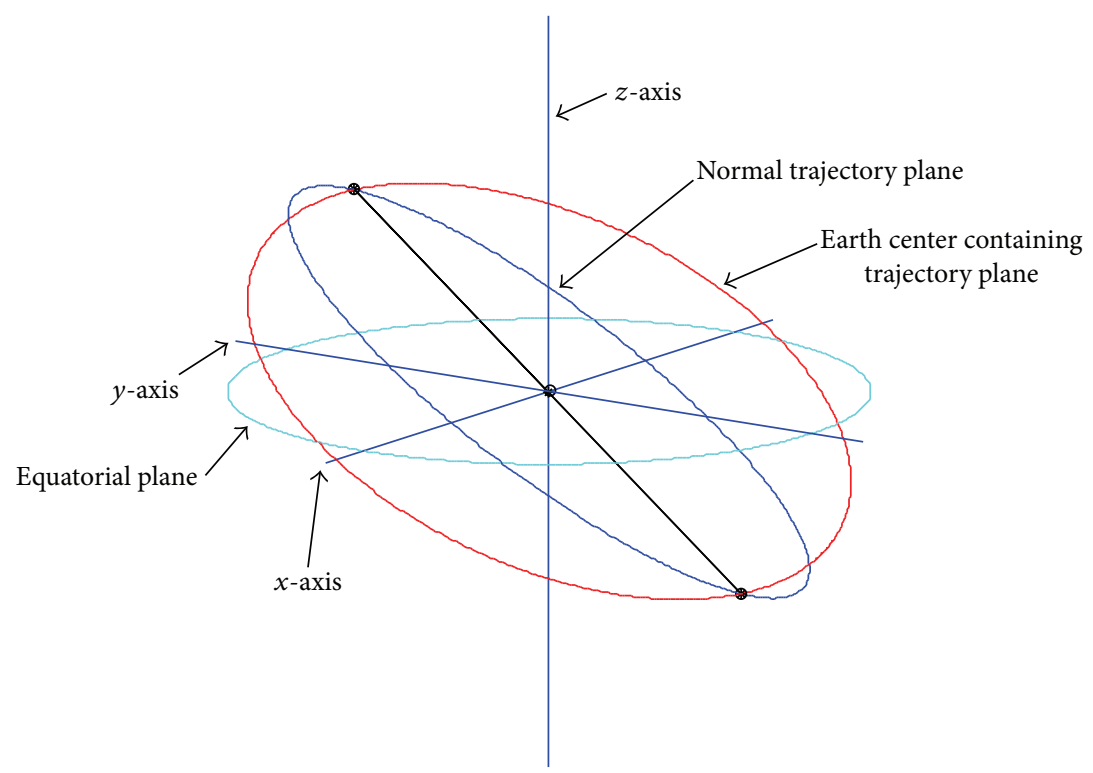

FIGURE 1: Ellipses pass through the target and launch points.

Using these relations, (16) can be written as

$$
\begin{aligned}
s_{1} n_{2} & =s_{2} n_{1}, \\
\frac{s_{1}}{n_{1}} & =\frac{s_{2}}{n_{2}}=k, \\
s_{1} & =n_{1} k, \\
s_{2} & =n_{2} k .
\end{aligned}
$$

Using (19) in (12), we have

$$
b^{2}\left(e_{1} n_{1}+e_{2} n_{2}\right) k=-a^{2} e_{3} s_{3} .
$$

Since $\widehat{n}$ is perpendicular to $\widehat{e}$, therefore

$$
\begin{aligned}
\widehat{n} \cdot \widehat{e} & =0, \\
n_{1} e_{1}+n_{2} e_{2} & =-n_{3} e_{3} .
\end{aligned}
$$

Using the above equation in (20), we have

$$
s_{3}=\frac{b_{2}}{a^{2}} n_{3} k
$$

Since center of the ellipse $\vec{s}=\left[s_{\overrightarrow{1}}, s_{2}, s_{3}\right]$ lies in the plane given by (1), therefore it should satisfy (1); that is,

$$
n_{1} s_{1}+n_{2} s_{2}+n_{3} s_{3}=d
$$

Using (19) and (22), the above equation becomes

$$
\begin{gathered}
n_{1}\left(n_{1} k\right)+n_{2}\left(n_{2} k\right)+n_{3}\left(\frac{b^{2}}{a^{2}} n_{3} k\right)=d, \\
k=\frac{d}{n_{1}^{2}+n_{2}^{2}+n_{3}^{2}\left(b^{2} / a^{2}\right)},
\end{gathered}
$$

since $\widehat{n}$ is a unit vector. Therefore

$$
n_{1}^{2}+n_{2}^{2}+n_{3}^{2}=1
$$

Using this equation in the above equation for $k$

$$
k=\frac{d}{1-\left(1-b^{2} / a^{2}\right) n_{3}^{2}},
$$

where $E$ is the eccentricity of the oblate spheroid. Substituting the value of $k$ in (19) and (26), the center of the ellipse is given by

$$
\begin{aligned}
\vec{s} & =\left[s_{1}, s_{2}, s_{3}\right] \\
& =\left[\frac{n_{1} d}{1-E^{2} n_{3}^{2}}, \frac{n_{2} d}{1-E^{2} n_{3}^{2}}, \frac{\left(b^{2} / a^{2}\right) n_{3} d}{1-E^{2} n_{3}^{2}}\right] .
\end{aligned}
$$

Special Case. If the plane given by (1) passes through the center of the oblate spheroid, then

$$
d=0
$$

and $\vec{s}=[0,0,0]$; that is, the center of the oblate spheroid will also be the center of the ellipse.

Semimajor Axis of the Ellipse. To find the semimajor axis of the ellipse, we take an arbitrary point $P(\vec{r})$ on the ellipse whose position vector $\vec{r}$ can be parameterized as

$$
\vec{r}=\vec{s}+\mu \widehat{e},
$$


where $\widehat{e}$ is the unit vector; this implies that

$$
\begin{gathered}
e_{1}^{2}+e_{2}^{2}+e_{3}^{2}=1, \\
\vec{r}=\left[s_{1}+\mu e_{1}, s_{2}+\mu e_{2}, s_{3}+\mu e_{3}\right] .
\end{gathered}
$$

Since point $P(\vec{r})$ also lies on the oblate spheroid, therefore it must satisfy (2):

$$
\begin{gathered}
{\left[\left(b^{2}\left(e_{1}^{2}+e_{2}^{2}\right)+a^{2} e_{3}^{2}\right)\right] \mu^{2}+2\left[\left(b^{2}\left(e_{1} s_{1}+e_{2} s_{2}\right)+a^{2} e_{3} s_{3}\right)\right] \mu} \\
+b^{2}\left(s_{1}^{2}+s_{2}^{2}\right)+a^{2} s_{3}^{2}-a^{2} b^{2}=0 .
\end{gathered}
$$

The roots of this equation (value of $\mu$ ) will have the same magnitude but opposite in sign; therefore, the coefficient of $\mu$ is zero. Thus, the above equation becomes

$$
\begin{aligned}
& {\left[b^{2}+\left(a^{2}-b^{2}\right) e_{3}^{2}\right] \mu^{2}+b^{2}\left(s_{1}^{2}+s_{2}^{2}\right)+a^{2} s_{3}^{2}-a^{2} b^{2}} \\
& \quad=0
\end{aligned}
$$

For particular, $\widehat{n}, \mu$ will be maximum and value of $\mu$ will be semimajor axis of an ellipse. To find the maximum value of $\mu$ with respect to $e_{3}$, we differentiate above equation with respect to $e_{3}$

$$
2\left[b^{2}+\left(a^{2}-b^{2}\right) e_{3}^{2}\right] \mu \frac{d \mu}{d e_{3}}+2 e_{3}\left(a^{2}-b^{2}\right) \mu^{2}=0
$$

and set

$$
\begin{gathered}
\frac{d \mu}{d e_{3}}=0, \\
2 e_{3}\left(a^{2}-b^{2}\right) \mu^{2}=0, \\
e_{3}=0 .
\end{gathered}
$$

Since $\hat{n}$ is perpendicular to $\hat{e}$, therefore

$$
n_{1} e_{1}+n_{2} e_{2}+n_{3} e_{3}=0 \text {. }
$$

Since $e_{3}=0$, this implies that

$$
\begin{aligned}
& e_{1}=n_{2} \alpha, \\
& e_{2}=-n_{1} \alpha .
\end{aligned}
$$

Since

$$
e_{1}^{2}+e_{2}^{2}+e_{3}^{2}=1
$$

this implies that

$$
\alpha=\frac{1}{\sqrt{n_{1}^{2}+n_{2}^{2}}} .
$$

Thus,

$$
\widehat{e}=\left[e_{1}, e_{2}, e_{3}\right]=\frac{1}{\sqrt{n_{1}^{2}+n_{2}^{2}}}\left[n_{2},-n_{1}, 0\right] .
$$

For caculated $\widehat{e}$, the maximum value of $\mu=a_{1}$ is

$$
\begin{gathered}
b^{2} a_{1}^{2}+b^{2}\left(s_{1}^{2}+s_{2}^{2}\right)+a^{2} s_{3}^{2}-a^{2} b^{2}=0, \\
a_{1}^{2}=a^{2}-\frac{a^{2}}{b^{2}} s_{3}^{2}=\left(s_{1}^{2}+s_{2}^{2}\right) .
\end{gathered}
$$

Using (27),

$$
a_{1}=a \sqrt{1-\frac{d^{2}}{a^{2}\left(1-E^{2} n_{3}^{2}\right)}} .
$$

This is the semimajor axis and the direction of the major axis is given by

$$
\widehat{e}=\frac{1}{\sqrt{n_{1}^{2}+n_{2}^{2}}}\left[n_{2},-n_{1}, 0\right] .
$$

Semiminor Axis of the Ellipse. Let $\widehat{u}$ be a unit vector along the minor axis of the ellipse; then

$$
\begin{aligned}
& \widehat{u} \cdot \widehat{e}=0, \\
& \widehat{u} \cdot \widehat{n}=0 .
\end{aligned}
$$

Thus, $\widehat{u}$ can be written as

$$
\begin{aligned}
& \widehat{u}=\widehat{e} \times \widehat{n}, \\
& \widehat{u}=\left[e_{2} n_{3},-e_{1} n_{3}, e_{1} n_{2},-e_{2} n_{1}\right] .
\end{aligned}
$$

Using (42) we have

$$
\widehat{u}=\frac{1}{\sqrt{n_{1}^{2}+n_{2}^{2}}}\left[-n_{1} n_{3},-n_{2} n_{3}, n_{1}^{2}+n_{2}^{2}\right] .
$$

If $b_{1}$ is the semiminor axis of the ellipse, then point $P(\vec{r})$ on the ellipse which is the closest to the center of the ellipse is given by

$$
\begin{aligned}
& \vec{r}=\vec{S}+b_{1} \widehat{u}, \\
& \vec{r}=\left[s_{1}+b_{1} u_{1}, s_{2}+b_{1} u_{2}+s_{3}+b_{1} u_{3}\right] .
\end{aligned}
$$

Since this point also lies on the oblate spheroid, therefore

$$
\frac{\left(s_{1}+b_{1} u_{1}\right)^{2}+\left(s_{2}+b_{1} u_{2}\right)^{2}}{a^{2}}+\frac{\left(s_{3}+b_{1} u_{3}\right)^{2}}{b^{2}}=1,
$$

$$
\begin{aligned}
& {\left[\left(b^{2}\left(u_{1}^{2}+u_{2}^{2}\right)+a^{2} u_{3}^{2}\right)\right] b_{1}^{2}} \\
& \quad+2\left[\left(b^{2}\left(u_{1} s_{1}+u_{2} s_{2}\right)+a^{2} u_{3} s_{3}\right)\right] b_{1} \\
& \quad+b^{2}\left(s_{1}^{2}+s_{2}^{2}\right)+a^{2} s_{3}^{2}-a^{2} b^{2}=0 .
\end{aligned}
$$

This equation is quadratic in $b_{1}$. Thus, the above equation should give us two values of $b_{1}$, both having the same 
magnitude but opposite in sign. It means that coefficient of $b_{1}$ in this equation should be zero. The above equation then becomes

$$
\begin{gathered}
{\left[\left(b^{2}\left(u_{1}^{2}+u_{2}^{2}\right)+a^{2} u_{3}^{2}\right)\right] b_{1}^{2}+b^{2}\left(s_{1}^{2}+s_{2}^{2}\right)+a^{2} s_{3}^{2}-a^{2} b^{2}=0} \\
b_{1}=b \frac{\sqrt{1-d^{2} / a^{2}-E^{2} n_{3}^{2}}}{1-E^{2} n_{3}^{2}}
\end{gathered}
$$

which is the semiminor axis of the ellipse. When the semimajor axis, semiminor axis, and center of the ellipse passing through the launch and target points are known, we can calculate the distance between launch and target points (which will be equal to the arc length between these two points of the ellipse) in the following manner. The equation of the ellipse having semimajor axis $a_{1}$ and semiminor axis $b_{1}$ is given by

$$
\frac{x^{2}}{a_{1}^{2}}+\frac{y^{2}}{b_{1}^{2}}=1
$$

The unit vectors along the major and minor axis are $\widehat{e}$ and $\widehat{u}$, respectively, and the centre of the ellipse with respect to Earth center Earth fixed frame (ECEF) is $\vec{s}=\left[s_{1}, s_{2}, s_{3}\right]$. Let $\vec{r}_{1}\left(x_{1}, y_{1}, z_{1}\right)$ be the position vector of launch point and let $\vec{r}_{2}\left(x_{2}, y_{2}, z_{2}\right)$ be the position vector of target point. Here $\phi_{1}$ is the geodetic latitude, $\lambda_{1}$ is the longitude of the launch point, $\phi_{2}$ is the geodetic latitude, and $\lambda_{2}$ is the longitude of the target point; then the Cartesian coordinates of the launch and target points in ECEF frame are

$$
\begin{aligned}
& x_{i}=a \cos u_{i} \cos \lambda_{i}, \\
& y_{i}=a \cos u_{i} \sin \lambda_{i}, \\
& z_{i}=b \sin u_{i}
\end{aligned}
$$

for $i=1,2$,

where $u_{1}$ and $u_{2}$ are reduced latitudes of the launch and target points, respectively, given by

$$
\tan u_{i}=\frac{b}{a} \tan \phi_{i}, \quad \text { for } i=1,2
$$

Also the position vectors $\vec{r}_{1}\left(x_{1}, y_{1}, z_{1}\right)$ and $\vec{r}_{2}\left(x_{2}, y_{2}, z_{2}\right)$ can be written as

$$
\vec{r}_{i}=\vec{s}+a_{i} \cos \theta_{i} \widehat{e}+b_{i} \sin \theta_{i} \widehat{u}, \quad \text { for } i=1,2,
$$

where $\theta_{1}, \theta_{2}$ are the reduced latitudes of launch and target points, respectively, with respect to ellipse:

$$
\begin{gathered}
\vec{r}_{1}-\vec{s}=a_{1} \cos \theta_{1} \widehat{e}+b_{1} \sin \theta_{1} \widehat{u}, \\
\widehat{u} \cdot\left(\vec{r}_{1}-\vec{s}\right)=b_{1} \sin \theta_{1}, \\
\widehat{e} \cdot\left(\vec{r}_{1}-\vec{s}\right)=a_{1} \cos \theta_{1} .
\end{gathered}
$$

Dividing (54) by (55), we have

$$
\begin{gathered}
\frac{b_{1}}{a_{1}} \tan \theta_{1}=\frac{\widehat{u} \cdot\left(\vec{r}_{1}-\vec{s}\right)}{\widehat{e} \cdot\left(\vec{r}_{1}-\vec{s}\right)}, \\
\tan \theta_{1}=\frac{a_{1} \widehat{u} \cdot\left(\vec{r}_{1}-\vec{s}\right)}{b_{1} \hat{e} \cdot\left(\vec{r}_{1}-\vec{s}\right)}, \\
\theta_{1}=\arctan \frac{a_{1} \widehat{u} \cdot\left(\vec{r}_{1}-\vec{s}\right)}{b_{1} \widehat{e} \cdot\left(\vec{r}_{1}-\vec{s}\right)} .
\end{gathered}
$$

Similarly

$$
\begin{gathered}
\tan \theta_{2}=\frac{a_{1} \widehat{u} \cdot\left(\vec{r}_{2}-\vec{s}\right)}{b_{1} \widehat{e} \cdot\left(\vec{r}_{2}-\vec{s}\right)}, \\
\theta_{2}=\arctan \frac{a_{1} \widehat{u} \cdot\left(\vec{r}_{2}-\vec{s}\right)}{b_{1} \widehat{e} \cdot\left(\vec{r}_{2}-\vec{s}\right)} .
\end{gathered}
$$

To calculate arc length of the ellipse given by (49) between $\theta_{1}$ and $\theta_{2}$, any point $r(x, y)$ on the ellipse can be written as

$$
\begin{aligned}
x & =a_{1} \cos \theta, \\
y & =b_{1} \sin \theta, \\
d x & =-a_{1} \sin \theta d \theta, \\
d y & =b_{1} \cos \theta d \theta .
\end{aligned}
$$

The arc length between $\theta_{1}$ and $\theta_{2}$ on the ellipse is given by

$$
S=\int_{\theta_{1}}^{\theta_{2}} \sqrt{a_{1}^{2} \sin ^{2} \theta+b_{1}^{2} \cos ^{2} \theta} d \theta
$$

which is the required distance between launch point and target point. There are two possible distances between $\theta_{1}$ and $\theta_{2}$ depending on the direction. In order to take the shorter distance, we replace the greater angle by $2 \pi-\theta_{i}(i=1$ or 2$)$ if the difference between $\theta_{1}$ and $\theta_{2}$ is greater than $\pi$.

\section{Numerical Simulations}

In numerical experimentation, we conducted several tests to calculate the effective ground range between launch and target points using oblate Earth model. There are infinite number of ellipses that can pass through target and launch points. So we need an additional information to fix the position of an ellipse. In articles $[1,2]$, the authors assumed that ellipse passes through the center of Earth but in reality the trajectory plane either contains the normal at the launch or target. If ellipse plane contains the normal at launch or target, there is no compulsion to contain the center of Earth. We will address the calculation of effective ground range for both the above-described conditions; namely, an ellipse passes through the center of Earth or from the normal at launch or target. In order to perform the ground range calculations, we fix the geocentric longitude and latitude of a launch site $\mathrm{A}\left(-100^{\circ}, 30^{\circ}\right)$ and change the location of target site. The effective ground ranges between launch 
TABLE 1: Comparison of ground ranges using oblate spheroid Earth model from a launch city A $\left(-100^{\circ}, 30^{\circ}\right)$.

\begin{tabular}{lccc}
\hline (Longitude, latitude) & Range-ND method $(\mathrm{km})$ & Range-MF method $(\mathrm{km})$ & Difference $(\mathrm{m})$ \\
\hline$\left(60^{\circ},-30^{\circ}\right)$ & 18104.8887315221 & 18105.1601439396 & 271.41241749996 \\
$\left(30^{\circ},-30^{\circ}\right)$ & 15252.3679549724 & 15252.4368861646 & 68.9311921942135 \\
$\left(75^{\circ},-30^{\circ}\right)$ & 19547.4843247251 & 19548.6946517136 & 1210.32698854469 \\
$\left(95^{\circ},-30^{\circ}\right)$ & 19066.0969508061 & 19066.7002432942 & 603.292488183797 \\
$\left(10^{\circ}, 25^{\circ}\right)$ & 10370.6552426009 & 10370.6407400275 & 14.5025733963848 \\
$\left(0^{\circ}, 0^{\circ}\right)$ & 10976.4405907028 & 10976.433648927 & 6.94177578952804 \\
$\left(-100^{\circ},-30^{\circ}\right)$ & 6677.23509886027 & 6677.23509886027 & $9.09494701772928 e-10$ \\
\hline
\end{tabular}

TABLE 2: Comparison of lengths of major axis using oblate spheroid Earth model from a launch city A $\left(-100^{\circ}, 30^{\circ}\right)$.

\begin{tabular}{lccc}
\hline $\begin{array}{l}\text { (Longitude, } \\
\text { latitude) }\end{array}$ & $\begin{array}{c}\text { Major axis length } \\
\text { ND method }(\mathrm{km})\end{array}$ & $\begin{array}{c}\text { Major axis length } \\
\text { MF method }(\mathrm{km})\end{array}$ & $\begin{array}{c}\text { Difference } \\
(\mathrm{m})\end{array}$ \\
\hline$\left(60^{\circ},-30^{\circ}\right)$ & 6378.14 & 6378.11 & 27.03 \\
$\left(30^{\circ},-30^{\circ}\right)$ & 6378.14 & 6378.11 & 25.84 \\
$\left(75^{\circ},-30^{\circ}\right)$ & 6378.14 & 6378.11 & 27.08 \\
$\left(95^{\circ},-30^{\circ}\right)$ & 6378.14 & 6378.11 & 27.11 \\
$\left(10^{\circ}, 25^{\circ}\right)$ & 6378.14 & 6378.12 & 19.67 \\
$\left(0^{\circ}, 0^{\circ}\right)$ & 6378.14 & 6378.11 & 26.93 \\
$\left(-100^{\circ}\right.$, & 6378.14 & 6378.14 & 0 \\
$\left.-30^{\circ}\right)$ & & & \\
\hline
\end{tabular}

and targets are calculated by using two methods ND method [2] and proposed MF method. In ND method, the elliptic plane passes through launch point, target point, and center of Earth and for MF method elliptic plane is defined by using the launch point, target point, and normal at the launch site which is normal to oblate spheroid Earth model. Table 1 shows the ranges difference in meters because the generated ellipses for both cases are different. One can expect the change range in couple of kilometers. We do emphasis the fact that the defining ellipse should contain the local normal at launch site or target site. The assumption that the elliptical plane should pass through the center of Earth provides good results but true condition generates slightly different values of effective ground range. Tables 2, 3, and 4 depict majors axis, minor axis, and center of ellipses for both methods. Clearly in our case, the intersecting ellipse has center different than oblate spheroid Earth model center which is $(0,0,0)$.

\section{Conclusion}

We have constructed explicitly the elements of intersecting ellipse, namely, the center, major, minor axis lengths, and their directions. The proposed method also provides the straight forward mechanism to plot the trace of intersecting ellipse on the oblate spheroid Earth model. The methods proposed in $[19,20]$ are the subcases of our developed method because ellipse always has to pass through launch and target sites and the third information one can define according to requirement which helps to define the normal to elliptical plane. The trajectory of ballistic missile lies in plane which contains the local normal to oblate spheroid Earth model
TABLE 3: Comparison of lengths of minor axis using oblate spheroid Earth model from a launch city A $\left(-100^{\circ}, 30^{\circ}\right)$.

\begin{tabular}{lccc}
\hline $\begin{array}{l}\text { Longitude, } \\
\text { latitude) }\end{array}$ & $\begin{array}{c}\text { Minor axis length } \\
\text { ND method }(\mathrm{km})\end{array}$ & $\begin{array}{c}\text { Minor axis length } \\
\text { MF method }(\mathrm{km})\end{array}$ & $\begin{array}{c}\text { Difference } \\
(\mathrm{m})\end{array}$ \\
\hline$\left(60^{\circ},-30^{\circ}\right)$ & 6372.65 & 6372.61 & 32.97 \\
$\left(30^{\circ},-30^{\circ}\right)$ & 6371.94 & 6371.92 & 26.73 \\
$\left(75^{\circ},-30^{\circ}\right)$ & 6372.76 & 6372.64 & 122.35 \\
$\left(95^{\circ},-30^{\circ}\right)$ & 6372.7 & 6372.66 & 37.71 \\
$\left(10^{\circ}, 25^{\circ}\right)$ & 6368.4 & 6368.3 & 96.41 \\
$\left(0^{\circ}, 0^{\circ}\right)$ & 6372.65 & 6372.56 & 90.3 \\
$\left(-100^{\circ}\right.$, & 6356.75 & 6356.75 & 0 \\
$\left.-30^{\circ}\right)$ & & & \\
\hline
\end{tabular}

TABLE 4: Center of elliptical arc.

\begin{tabular}{lccc}
\hline $\begin{array}{l}\text { Longitude, } \\
\text { latitude })\end{array}$ & $\begin{array}{c}x \text {-Coordinate } \\
(\mathrm{km})\end{array}$ & $\begin{array}{c}y \text {-Coordinate } \\
(\mathrm{km})\end{array}$ & $\begin{array}{c}z \text {-Coordinate } \\
(\mathrm{km})\end{array}$ \\
\hline$\left(60^{\circ},-30^{\circ}\right)$ & -2.88 & -8.97 & -15.95 \\
$\left(30^{\circ},-30^{\circ}\right)$ & -5.49 & -8.09 & -15.24 \\
$\left(75^{\circ},-30^{\circ}\right)$ & -0.61 & -9.38 & -15.98 \\
$\left(95^{\circ},-30^{\circ}\right)$ & -0.89 & -9.35 & -16 \\
$\left(10^{\circ}, 25^{\circ}\right)$ & 7.01 & -8.13 & -11.61 \\
$\left(0^{\circ}, 0^{\circ}\right)$ & 0.05 & -9.45 & -15.89 \\
$\left(-100^{\circ}\right.$, & 0 & 0 & 0 \\
$\left.-30^{\circ}\right)$ & 0 & & \\
\hline
\end{tabular}

either at launch site or target site. The trajectory plane which passes through the center of Earth, generally, does not contain normal at launch site or target site. We have shown that our numerical simulations provide the effective ground ranges in the case of normal trajectory plane which are different in couple of kilometers from Earth center passing trajectory plane.

\section{Conflict of Interests}

The authors declare that there is no conflict of interests regarding the publication of this paper.

\section{Acknowledgments}

This work was funded by the Deanship of Scientific Research (DSR), King Abdulaziz University, Jeddah, under Grant no. 
(130-098-D1434). The authors, therefore, acknowledge with thanks DSR technical and financial support.

\section{References}

[1] F. A. Abd El-Salam and S. E. Abd El-Bar, "Computation of the different errors in the ballistic missiles range," ISRN Applied Mathematics, vol. 2011, Article ID 349737, 16 pages, 2011.

[2] J. A. Isaacson and D. R. Vaughan, Estimation and Prediction of Ballistic Missile Trajectories, RAND, Santa Monica, Calif, USA, 1996.

[3] W. J. Harlin and D. A. Cicci, "Ballistic missile trajectory prediction using a state transition matrix," Applied Mathematics and Computation, vol. 188, no. 2, pp. 1832-1847, 2007.

[4] A. Akgül and S. Karasoy, "Development of a tactical ballistic missile trajectory prediction tool," Istanbul University-Journal of Electrical and Electronics Engineering, vol. 5, no. 2, pp. 14631467, 2005.

[5] N. X. Vinh, P. T. Kabamba, and T. Takehira, "Optimal interception of a maneuvering long-range missile," Acta Astronautica, vol. 48, no. 1, pp. 1-19, 2001.

[6] S. A. Kamal, "Cross range error in the lambert scheme," in Proceedings of the 10th National Aeronautical Conference, S. R. Sheikh, Ed., pp. 255-263, College of Aeronautical Engineering, PAF Academy, Risalpur, Pakistan, April 2006.

[7] S. Bhowmik and C. Sadhukhan, "Application of extended kalman filter to tactical ballistic missile re-entry problem," 2007, http://arxiv.org/abs/0707.1918.

[8] S. A. Kamal, "The multi-stage-lambert scheme for steering a satellite-launch vehicle (SLV)," in Proceedings of the 12th IEEE International Multitopic Conference (INMIC '08), M. K. Anisx, M. K. Khan, and S. J. H. Zaidi, Eds., pp. 294-300, Bahria University, Karachi, Pakistan, December 2008.

[9] C. Y. Liu and C. T. Chen, "Tracking the warhead among objects separation from the reentry vehicle in a clear environment," Defence Science Journal, vol. 59, no. 2, pp. 113-125, 2009.

[10] M. Z. Tidrow and W. R. Dyer, "Infrared sensors for ballistic missile defense," Infrared Physics and Technology, vol. 42, no. 35, pp. 333-336, 2001.

[11] U. K. Singha, V. Padmanabhanb, and A. Agarwalb, "Dynamic classification of ballistic missiles using neural networks and hidden Markov models," Applied Soft Computing, vol. 19, pp. 280-289, 2014.

[12] J.-H. Jung, K.-T. Kim, S.-H. Kim, and S.-H. Park, "Microdoppler extraction and analysis of the ballistic missile using rda based on the real flight scenario," Progress in Electromagnetics Research M, vol. 37, pp. 83-93, 2014.

[13] G. Spinardi, "Technical controversy and ballistic missile defence: disputing epistemic authority in the development of hit-to-kill technology," Science as Culture, vol. 23, no. 1, pp. 126, 2014.

[14] G. Spinardi, "Technical controversy and ballistic missile defence: disputing epistemic authority in the development of hit-to-kill technology," Science as Culture, vol. 23, no. 1, pp. 126, 2014.

[15] W. Hong, C. M. Tong, and J. J. Sun, "Study on precessional motion simulation of ballistic missile midcourse," Applied Mechanics and Materials, vol. 484, pp. 1087-1092, 2014.

[16] A. J. Prakash, M. Raja, S. Gupta et al., "Stability analysis of autopilot systems for ballistic missile using MATLAB," in
Proceedings of the Conference on Advances in Communication and Control Systems, Atlantis Press, 2013.

[17] R. Bate Roger, D. D. Mueller, and J. E. White, Fundamentals of Astrodynamics: (Dover Books on Physics), Dover, 2013.

[18] N. Prabhakar, I. D. Kumar, S. K. Tata, and V. Vaithiyanathan, "A simplified guidance for target missiles used in ballistic missile defence evaluation," Journal of The Institution of Engineers (India): Series C, vol. 94, no. 1, pp. 31-36, 2013.

[19] P. R. Escobal, "Calculation of the surface range of a ballistic missile," AIAA Journal, vol. 2, no. 3, pp. 571-573, 1964.

[20] B. U. Nguyen and M. E. Dixson, "Computation of effective ground range using an oblate Earth model," The Journal of the Astronautical Sciences, vol. 51, no. 3, pp. 291-305, 2003. 


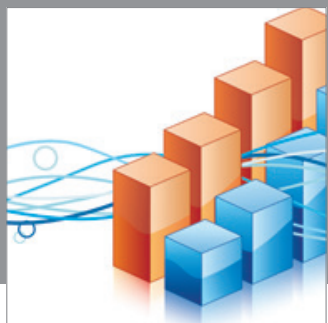

Advances in

Operations Research

mansans

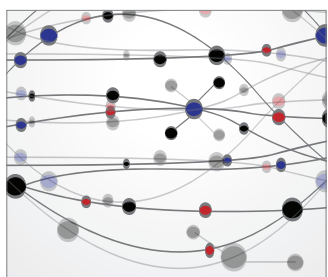

The Scientific World Journal
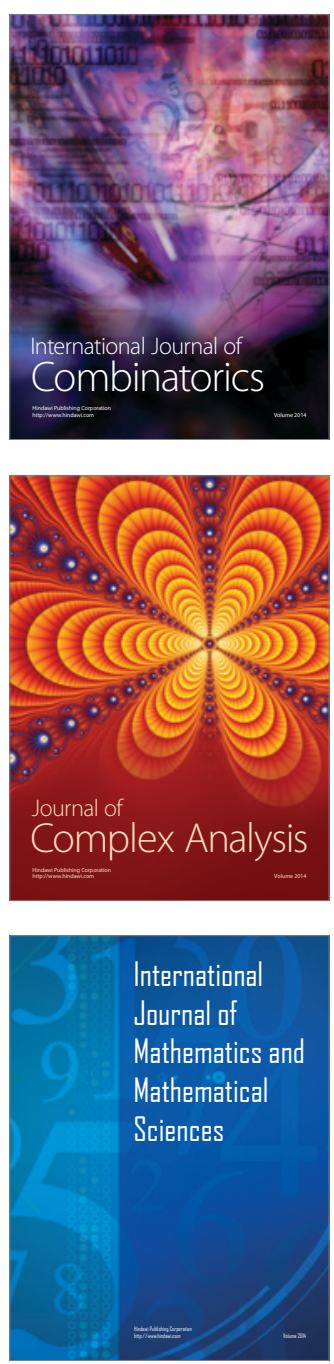
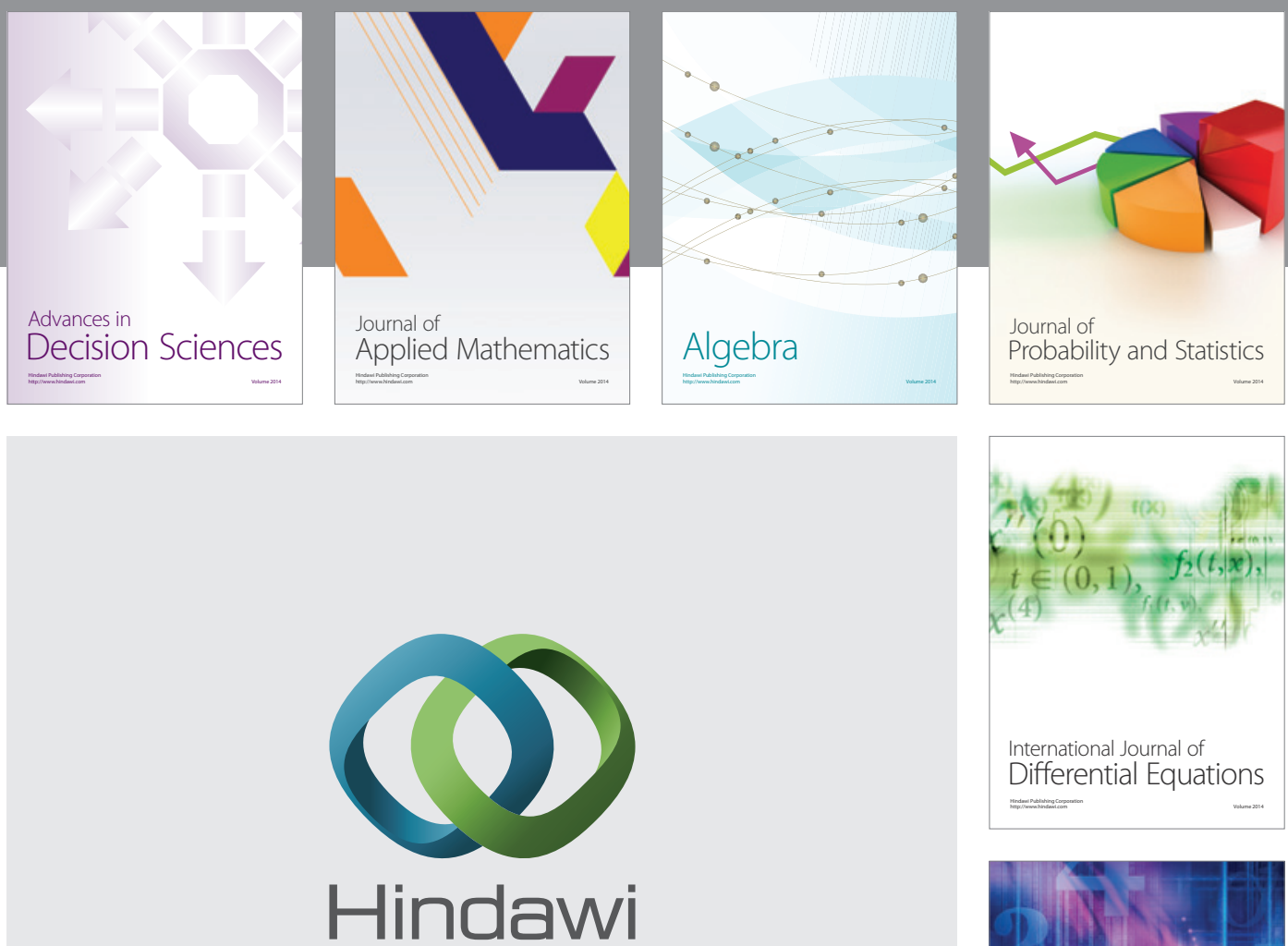

Submit your manuscripts at http://www.hindawi.com
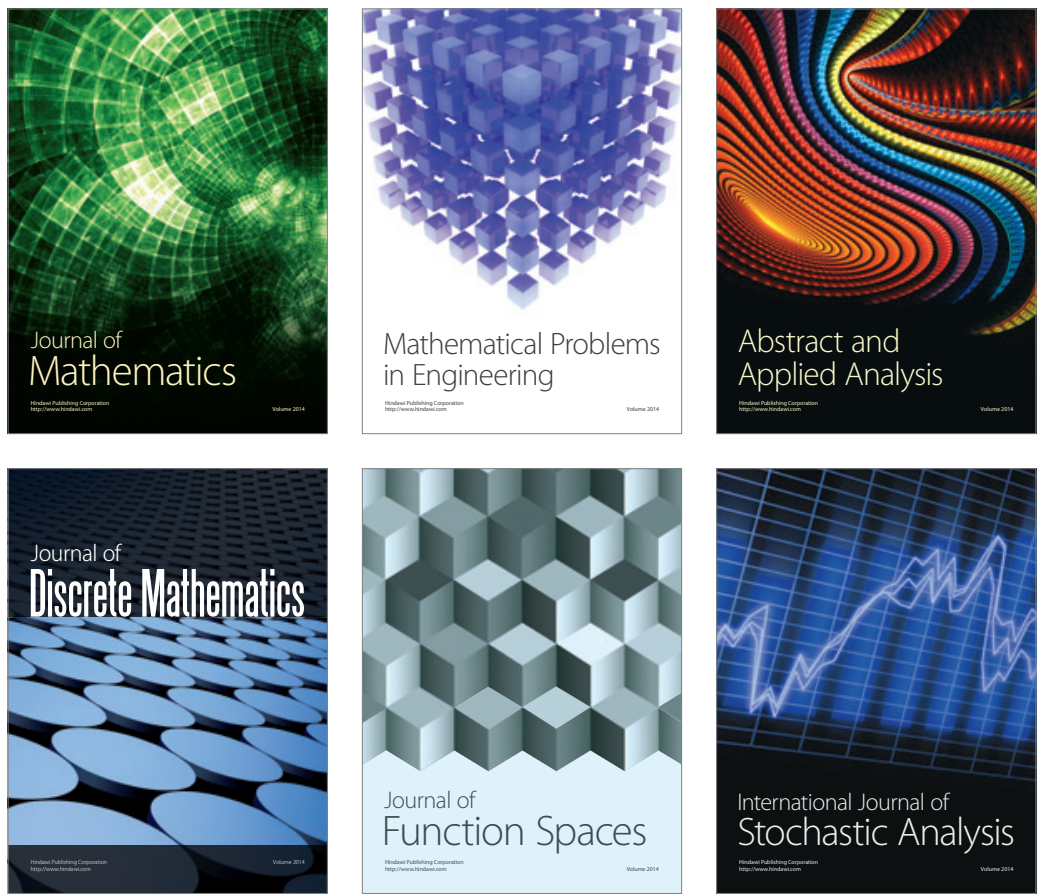

Journal of

Function Spaces

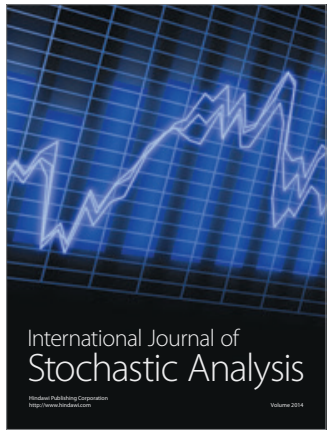

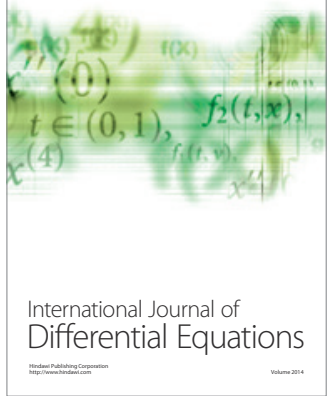
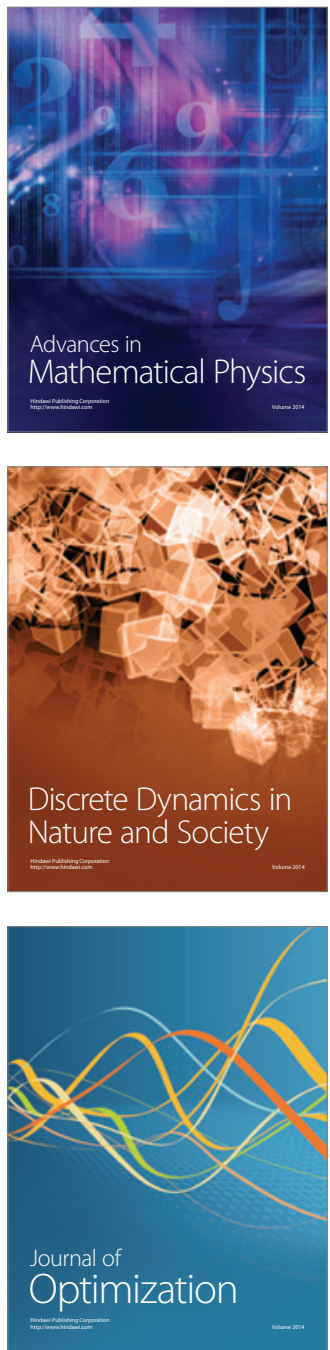